\title{
Light trapping and near-unity solar absorption in a three-dimensional photonic-crystal
}

\author{
Ping Kuang, ${ }^{1}$ Alexei Deinega, ${ }^{2}$ Mei-Li Hsieh, ${ }^{3}$ Sajeev John, ${ }^{2}$ and Shawn-Yu Lin ${ }^{1, *}$ \\ ${ }^{1}$ The Future Chips Constellation and the Department of Physics, Applied Physics, and Astronomy, \\ Rensselaer Polytechnic Institute, 110 8th Street, Troy, New York 12180, USA \\ ${ }^{2}$ Department of Physics, University of Toronto, 60 St. George Street, Toronto, Ontario M5S 1A7, Canada \\ ${ }^{3}$ Department of Photonics, National Chiao-Tung University, Hsinchu, Taiwan \\ ${ }^{*}$ Corresponding author: sylin@rpi.edu
}

Received July 25, 2013; accepted September 2, 2013;

posted September 10, 2013 (Doc. ID 194567); published October 14, 2013

\begin{abstract}
We report what is to our knowledge the first observation of the effect of parallel-to-interface-refraction (PIR) in a three-dimensional, simple-cubic photonic-crystal. PIR is an acutely negative refraction of light inside a photoniccrystal, leading to light-bending by nearly 90 deg over broad wavelengths $(\lambda)$. The consequence is a longer path length of light in the medium and an improved light absorption beyond the Lambertian limit. As an illustration of the effect, we show near-unity total absorption $(\geq 98 \%)$ in $\lambda=520-620 \mathrm{~nm}$ and an average absorption of $\sim 94 \%$ over $\lambda=400-700 \mathrm{~nm}$ for our $\alpha$-Si:H photonic-crystal sample of an equivalent bulk thickness of $\tilde{t}=450 \mathrm{~nm}$. Furthermore, we have achieved an ultra-wide angular acceptance of light over $\theta=0^{\circ}-80^{\circ}$. This demonstration opens up a new door for light trapping and near-unity solar absorption over broad $\lambda$ s and wide angles. @ 2013 Optical Society of America

OCIS codes: (350.6050) Solar energy; (050.5298) Photonic crystals.

http://dx.doi.org/10.1364/OL.38.004200
\end{abstract}

There is a great deal of interest in efficient light trapping in thin film solar cells for approaching the ShockleyQueisser limit [1] and for low cost photovoltaics. As solar radiation is broadband [2] and its incident angle on earth varies over time, solar light trapping must be effective over a broad range of wavelengths $(\lambda)$ and wide angles $(\theta)$. A random surface-structure can be used to trap light by multiple scatterings and leads to a prolonged optical path. For a properly prepared random surface, geometric optics predicts an increased path length by as much as $4 n^{2}$ [3]. However, the geometric optics approach is less effective where the sample thickness is comparable to the incident $\lambda$ of light. Accordingly, sub- $\lambda$ surface-structures, including plasmonic, nano-wire, and nano-cone, have been proposed as new routes for light trapping [ $\underline{4}-\underline{9}$ ]. Periodic surface-structures, such as one- and twodimensional photonic-crystals [10-13], have also been studied due to their ability to localize and trap light near the band edge. Most recently, a three-dimensional (3D) simple-cubic photonic-crystal has been proposed to trap light by anomalous light refraction [14-16] parallel-to-theinterface (PIR) of a thin film sample [16-18]. This extreme light-bending by nearly $90 \mathrm{deg}$ is expected to offer a two orders of magnitude absorption enhancement over that of single-path absorption. So far, there is no experimental demonstration of enhanced solar absorption over broad $\lambda$ s and wide $\theta$ s using $3 \mathrm{D}$ photonic-crystal thin film architecture.

Our 3D photonic-crystal possesses simple-cubic symmetry. For two decades, the most studied 3D photonic-crystal has a diamond symmetry that produces a large photonic bandgap [19]. Here, we choose simplecubic symmetry to enforce negative refraction in the photonic-allowed bands. One experimental realization of the lattice structure is to stack layers of 1D-gratings in a sequential fashion [19] as shown in Fig. 1(a). Two stacking layers form one unit-cell of the simple-cubic lattice. The vertical and horizontal red arrows indicate the directions of the incident and refracted light, respectively. In Fig. 1(c), we show the corresponding Brillouin zone and the symmetry points in wave vector space.

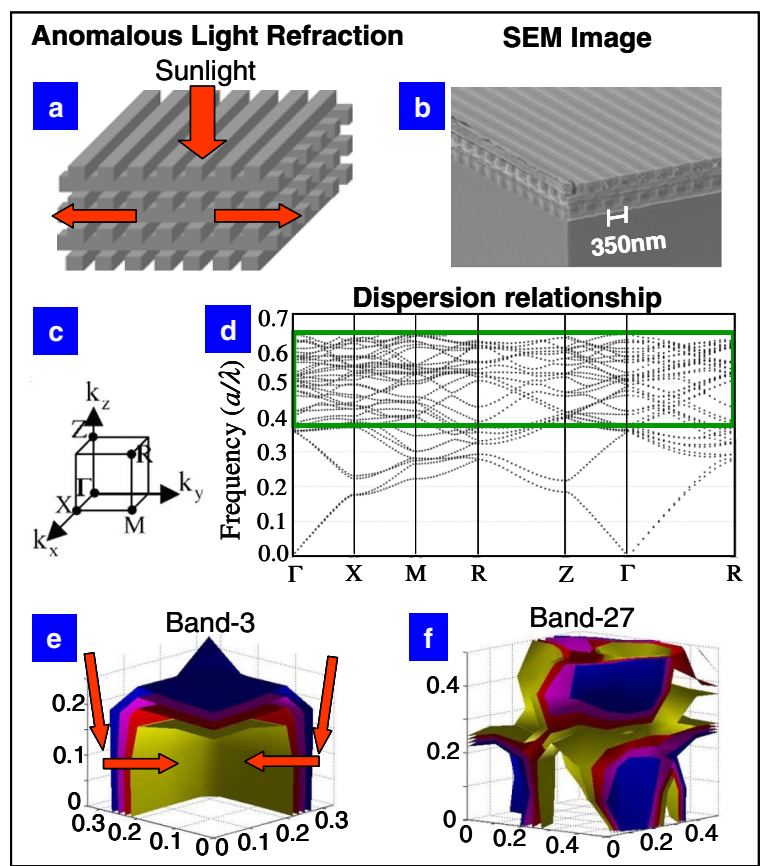

Fig. 1. Anomalous light refraction in a $3 \mathrm{D}$ photonic-crystal. (a) Schematic of a simple-cubic lattice structure. The direction of light refraction is indicated as red arrows. (b) SEM image of the fabricated sample with a lattice constant of $a=350 \mathrm{~nm}$. (c) The corresponding first Brillouin zone in $k$-space. (d) The calculated dispersion, frequency versus wave vector, of the lattice. The green square box corresponds to wavelengths of $\lambda \approx 450-800 \mathrm{~nm}$. (e) An iso-frequency plot of band-3. It has a square-like contour, allowing light refraction PIR. The red arrows indicate the flow of energy. (f) An iso-frequency plot of band-27, which is more complicated but still exhibits a square-like contour. 
In Fig. 1(d), we show the computed dispersion, frequency versus wave vector, of our $3 \mathrm{D}$ photonic-crystal sample. For a sample with lattice constant $a=300 \mathrm{~nm}$, the green square box corresponds to allowed bands in the relevant solar wavelength of $\lambda=450-800 \mathrm{~nm}$. In Fig. 1(e), we show an iso-frequency plot of band-3 of the dispersion shown in Fig. 1(d). It has a square-like contour, reflecting the simple-cubic symmetry and also allowing for light refraction parallel to the interface. The red arrows indicate the flow of energy, which is along the gradient of the constant frequency contour. In Fig. 1(f), we show an iso-frequency plot of band-27, which is slightly more complicated but still exhibits a square-like contour as well as the effect of PIR. We comment that, for higher-order allowed bands, such as band27 , the optical modes are more densely populated and the high density-of-states (DOS) regime has a nearly continuous spectrum. On the other hand, for those lower-order bands such as band-3, the bands are much more discrete and the peaks in DOS become separated. This contrast in the DOS spectrum will have important consequences on the absorption spectrum of our simple-cubic lattice.

Our 3D simple-cubic lattice was fabricated using a standard microelectronic process over a 4-in. wafer $[19,20]$. In the first step, hydrogenated amorphous silicon $(\alpha-\mathrm{Si}: \mathrm{H})$ is deposited on a fused silica wafer. The $1 \mathrm{D}$ grating is created by deep UV lithography and the grating patterns then transferred to the $\alpha$-Si:H thin film by deep reactive-ion-beam [21]. After removal of the resist, hydrogen silsesquioxane (HSQ) is spin-coated onto the pattern for planarization. After thermal curing, excess HSQ overlayer is removed and a one-layer grating is created. By repeating the process three more times, a 4-layer simple-cubic lattice is fabricated. In Fig. 1(b), we show a scanning electron micrograph of a successfully fabricated 4-layer sample. It has a lattice constant of $a=350 \mathrm{~nm}$, rod-width (and height) of $w=a / 2=175 \mathrm{~nm}$.

In Figs. 2(a)-2(c), we show spectra taken from an unstructured $\alpha$-Si:H thin film ( $t=600 \mathrm{~nm}$ thick) and two photonic-crystals with $a=350$ and $450 \mathrm{~nm}$, respectively. The spectra include total transmission (blue curve), reflection (red curve), and absorption (green curve) and were measured using an integrating sphere setup. Computational results are also shown as dashed lines. In Fig. 2(a), the thin film has an intermediate absorption of $A \approx 60 \%$ in $400 \mathrm{~nm} \leq \lambda \leq 580 \mathrm{~nm}$ and a weak absorption of $A \leq 30 \%$ in $640 \mathrm{~nm} \leq \lambda \leq 800 \mathrm{~nm}$ (the gray region). In the gray region, the weak absorption is due to the fact that it is near the $\alpha$-Si:H bandgap of $\lambda_{g} \approx 700 \mathrm{~nm}$. Meanwhile, the strong oscillations in both the transmission and reflection spectra are due to multiple interferences $[16,17]$. This is the region where strong absorptionenhancement is needed. In Fig. 2(b), the $a=350 \mathrm{~nm}$ photonic-crystal shows a higher absorption of $A \approx 85 \%$ over a broader range in $400 \mathrm{~nm} \leq \lambda \leq 630 \mathrm{~nm}$. The weakly absorbing region of $A \leq 30 \%$ (the gray region) now moves to $680 \mathrm{~nm} \leq \lambda \leq 800 \mathrm{~nm}$. This enhanced absorption is accomplished with a $4 \mathrm{~L}$ photonic-crystal having a $50 \%$ fillingfraction and an equivalent bulk thickness of $\tilde{t}=350 \mathrm{~nm}$. Note that there are also resonant oscillations $[16,17]$ in longer $\lambda \mathbf{s}(\lambda \geq 700 \mathrm{~nm})$, or the lower-order allowed bands in the dispersion shown in Fig. 1(c). We further
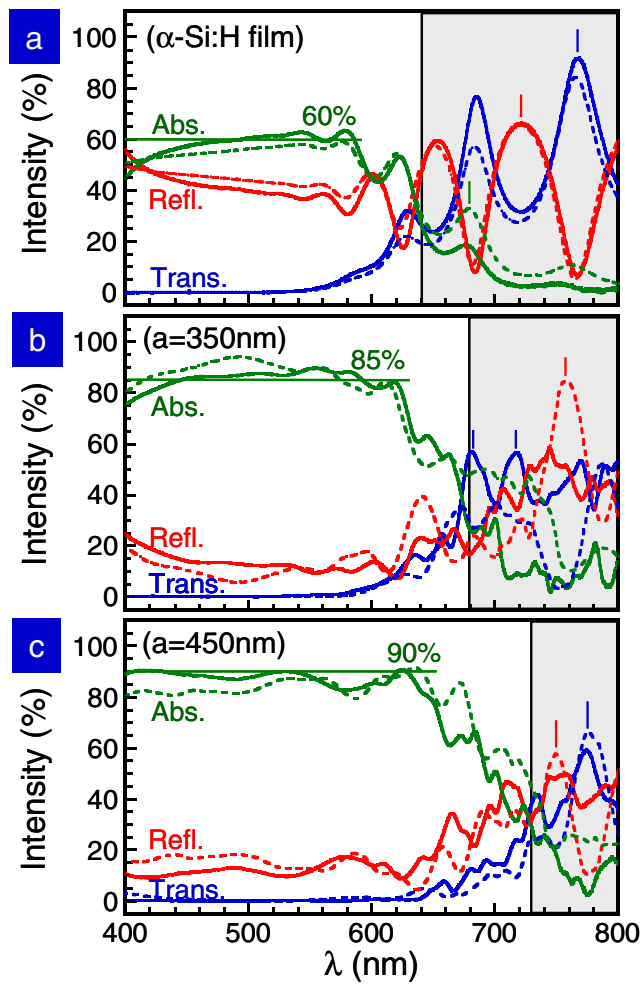

Fig. 2. Measured and computed total transmission, reflection, and absorption spectra. (a) The measured spectra for a $600 \mathrm{~nm}$ thick $\alpha$-Si:H thin film, showing an absorption of $A \approx 60 \%$ in $400 \mathrm{~nm} \leq \lambda \leq 580 \mathrm{~nm}$. In the gray region, the data shows a weak absorption $(A \leq 30 \%)$ and also resonant oscillations in both the transmission and reflection spectra. (b) The $a=350 \mathrm{~nm}$ photonic-crystal sample shows a higher absorption of $A \approx 85 \%$ in $400 \mathrm{~nm} \leq \lambda \leq 630 \mathrm{~nm}$. (c) The $a=450 \mathrm{~nm}$ photoniccrystal shows an even higher absorption of $A \sim 90 \%$ in $400 \mathrm{~nm} \leq \lambda \leq 640 \mathrm{~nm}$.

note that the experimental and computational results agree well. Finally, in Fig. 2(c), $a=450 \mathrm{~nm}$ photoniccrystal shows an even higher absorption of $A \sim 90 \%$ in $400 \mathrm{~nm} \leq \lambda \leq 640 \mathrm{~nm}$. The weakly absorbing region moves further to $725 \mathrm{~nm} \leq \lambda \leq 800 \mathrm{~nm}$ (the gray region). Again, the agreement between experimental and computational results is good.

There are two points worth noting. First, in the range $\lambda=400-650 \mathrm{~nm}$, the increase of absorption from $A \approx 60 \%$ to $85 \%-90 \%$ indicates a significant enhancement to absorption over planar film of similar thickness by the simple-cubic lattice. To reach near-unity absorption, the remaining $10 \%$ is lost mainly to reflection, which can be eliminated by a proper antireflective coating [22]. Second, the systematic shift of the weakly absorbing region (the gray region) to longer $\lambda$, i.e., from $\lambda=580$, 680 to $725 \mathrm{~nm}$, shows that absorption enhancement depends on the lattice constant. This dependence can be understood by observing scaling of the photoniccrystal dispersion with its lattice constant. This scaling dependence suggests that the absorption enhancement may originate from the engineered photonic-crystal dispersion, exhibiting a PIR effect.

To better quantify the absorption enhancement, we define an "enhancement factor" [7] as the ratio of absorption between a simple-cubic lattice and an unstructured 
$600 \mathrm{~nm}$ thick $\alpha$-Si:H thin-film. In Fig. 3(a), we show the enhancement factor as a function of $\lambda$ for three simple-cubic samples: $a=350 \mathrm{~nm}$ (red curve), $400 \mathrm{~nm}$ (green curve), and $450 \mathrm{~nm}$ (blue curve). As a benchmark, the maximum enhancement (black dotted curve) is needed to reach perfect absorption (100\%) and is also calculated from Fig. 2(a) data. In $400 \mathrm{~nm} \leq \lambda \leq 600 \mathrm{~nm}$, the observed enhancement factor approaches the maximum value of 2 for all three samples. In this regime, $\alpha$-Si:H material has a strong absorption constant and there is less need for strong enhancement. In the longer $\lambda \mathbf{s}$, $600 \mathrm{~nm} \leq \lambda \leq 800 \mathrm{~nm}$, the enhancement factor exhibits discrete oscillations and also higher values for those larger lattice constant samples. The discrete oscillations are attributed to resonances due to PIR at the lower-order allowed bands of the dispersion shown in Fig. 1(c). The stronger enhancement at larger lattice constants is caused by movement of the high DOS regime in the dispersion toward longer $\lambda \mathrm{s}$. For a sample where $a=450 \mathrm{~nm}$ $(t=450 \mathrm{~nm})$, the enhancement is 17 times, or $\lambda=$ $720 \mathrm{~nm}$ over that for the thin film $(t=600 \mathrm{~nm})$. The enhancement may be further increased to 50 times at $\lambda \approx$ $780 \mathrm{~nm}$ by using a back-reflector. This value is already close to the predicted two orders of magnitude enhancement in 3D photonic-crystal structures [7,16,17].

In essence, the phenomenon of PIR is a result of the intricate dispersion engineering of a $3 \mathrm{D}$ photonic-crystal. Theoretically, the absorption enhancement due to the PIR effect must follow the dispersion and scale of the lattice constant for a photonic-crystal. In the following, we study the lattice constant dependence of absorption in simple-cubic lattices. We assume no reflection from the structures and examine the absorption (Abs) over (1-R) ratio, which accounts for the incident light that enters the air/structure interface.

The Abs/(1-R) ratio is shown in Fig. 3(b). In the range $400 \mathrm{~nm} \leq \lambda \leq 600 \mathrm{~nm}$, the ratio is close to unity and, hence, all the light that enters the structure was absorbed. In the range $600 \mathrm{~nm} \leq \lambda \leq 750 \mathrm{~nm}$, the absorption curve shows a rapid drop and a clear lattice-constant dependence. Specifically, as the lattice-constant is increased from $a=350,400$ to $450 \mathrm{~nm}$, the drop to $50 \% \mathrm{ab}-$ sorption (the horizontal line) is extended to longer $\lambda \mathbf{s}$, from $\lambda=670,700$ to $730 \mathrm{~nm}$ (the vertical arrows). We comment that the PIR effect arises when electromagnetic dispersions are "flat" in the photonic allowed bands [16]. As the lattice-constant increases, the band-edge also shifts to longer- $\lambda \mathrm{s}$, allowing more resonance modes for lighttrapping and guiding. This point follows from the scaling property of photonic-crystal dispersion. The scaling confirms our earlier assertion that the absorption enhancement is a direct consequence of dispersion engineering of a simple-cubic lattice. Furthermore, the Lambertian limit is calculated using optical constants measured by ellipsometry, shown in Fig. 3(c). The measured optical constants are also independently verified by using polarizer-sample-analyzer ellipsometry [23]. The data show that all three samples exceed the statistical raytrapping limit (the Lambertian limit) for a broad $\lambda$ range $700 \mathrm{~nm} \leq \lambda \leq 800 \mathrm{~nm}$. For comparison, the Lambertian limit is also calculated with optical constants obtained by Fujiwara [24] and the samples exceed the limit in $760 \mathrm{~nm} \leq \lambda \leq 800 \mathrm{~nm}$.

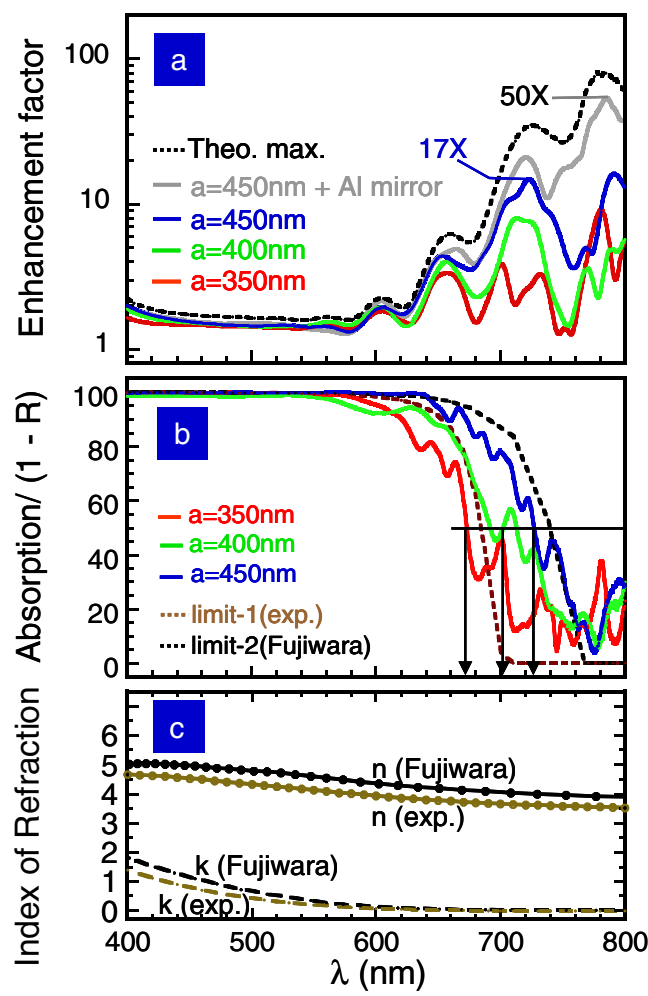

Fig. 3. Enhancement factor and broadband absorption. (a) Enhancement of absorption of the $4 \mathrm{~L}$ simple-cubic samples over the $600 \mathrm{~nm} \alpha$-Si:H thin film. The dotted line represents the maximum enhancement needed to reach $100 \%$ absorption. (b) Total absorption/(1-R) plot, showing that the absorption edge (the vertical arrows) is systematically shifted to longer $\lambda \mathrm{s}$ as the lattice constant is increased. The light-gray and dark-gray dotted lines are the Lambertian limits, calculated using our measured optical constants and those taken from the literature, respectively. (c) Plot of optical constants measured from $\alpha$-Si:H thin film (light-gray dots) and those taken from the literature (darkgray dots).

To further improve the absorption, we now introduce a back-reflector and an antireflection-coating into our photonic-crystal structures. In Fig. 4(a), we show absorption spectra taken from four different samples. The two spectra for $a=350 \mathrm{~nm}$ (red curve) and $450 \mathrm{~nm}$ (blue curve) serve as references. The blue dashed and black dotted curves are data taken from samples with an aluminum back-reflector and with both $\mathrm{Al}$ reflector/ antireflection coating, respectively. The combination of photonic-crystal and aluminum reflector yields an average total absorption of $\sim 90 \%$ over $400 \mathrm{~nm} \leq \lambda \leq 700 \mathrm{~nm}$ and $\sim 50 \%$ over $700 \mathrm{~nm} \leq \lambda \leq 800 \mathrm{~nm}$. By adding an aluminum reflector and antireflection coating [25], an average total absorption of $94 \%$ over $400 \mathrm{~nm} \leq \lambda \leq 700 \mathrm{~nm}$ is obtained. Meanwhile, near-unity total absorption ( $\geq 98 \%)$ in $\lambda=520-620 \mathrm{~nm}$ is achieved for our 4L simplecubic lattice. From the absorption data, and assuming that for each absorbed photon an electron-hole pair is generated and collected, the maximum achievable photocurrent density (MAPD) can be computed [7]. For the $\alpha$-Si:H thin film and $a=350 \mathrm{~nm}$ and $450 \mathrm{~nm}$ photoniccrystal samples, the calculated MAPDs are 9.1, 15.0, and $17.2 \mathrm{~mA} / \mathrm{cm}^{2}$, respectively. Hence, using photoniccrystals alone could double the useful electrical current 

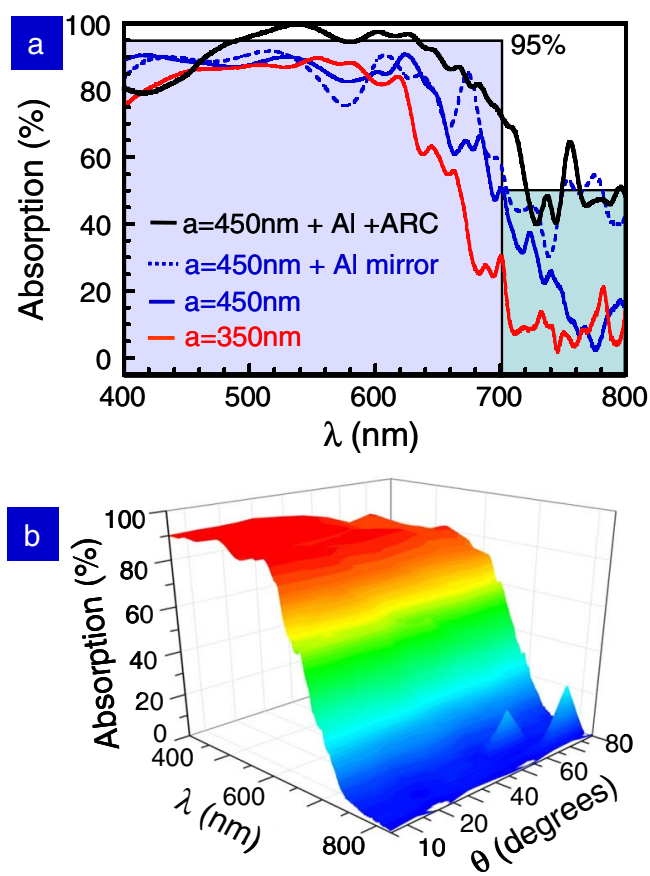

Fig. 4. Near-unity absorption and broad acceptance angle. (a) Comparison of measured absorption data taken from $a=$ 350 and $450 \mathrm{~nm}$ samples, $a=450 \mathrm{~nm}$ sample with an $\mathrm{Al}$ reflector and also $a=450 \mathrm{~nm}$ sample with an Al-reflector and antireflection coating. (b) $3 \mathrm{D}$ plot of the measured absorption versus $\lambda$ and incident angle $(\theta)$ for a $4 \mathrm{~L}$ simple-cubic photonic-crystal sample.

generated. Furthermore, by adding an aluminum reflector and Al-reflector/antireflection coating combination, the MAPDs are increased to 19.1 and $20.2 \mathrm{~mA} / \mathrm{cm}^{2}$, respectively. We note that there is a pathway to conformably coat the 3D simple-cubic lattice with a p-i-n junction for solar absorption and photocurrent generation [26].

Last, one unique feature of the PIR effect in a 3D simple-cubic lattice, is its ability to achieve high absorption over broad angles of incidence $\theta$. It has been theoretically predicted that, for a wide range of off-normal incident wave vectors, the propagation directions of the Bloch modes inside the lattice are parallel to the air/lattice interface, resulting in high absorption for broad $\theta \mathbf{s}[13,16]$. To validate such an effect experimentally and to further confirm the PIR effect, the $\theta$-dependence of absorption was measured for our sample, with a lattice constant $a=450 \mathrm{~nm}$. The total absorption measurements were taken with $\theta$ ranging from $5^{\circ}$ up to $80^{\circ}$ (in $10^{\circ}$ increments). The results are shown in the surface plot in Fig. 4(b). The plot shows the absorption is sustained at around $90 \%$ in the shorter $\lambda$ range, up to $50^{\circ}$. It is only reduced to $80 \%$ absorption at $\theta=70^{\circ}$. At $\theta=80^{\circ}$, the absorption is still around 70\%. Furthermore, in longer $\lambda$ ranges $(\lambda>600 \mathrm{~nm})$, the drop in absorption is much less noticeable. This indicates that high DOS resonance modes are maintained by the PIR effect of the 3D photonic-crystal thin film architecture even at very high $\theta$ s in the longer $\lambda$ range.

In summary, we report what is to our knowledge the first observation of the PIR effect in a 3D simple-cubic photonic-crystal. Specifically, an average absorption of $94 \%$ in $\lambda=400-700 \mathrm{~nm}$ is shown for the $4 \mathrm{~L}$ photonic- crystal with an equivalent bulk thickness of $\tilde{t}=450 \mathrm{~nm}$. Near-unity total absorption $(\geq 98 \%)$ is achieved in $\lambda=520-620 \mathrm{~nm}$. We have also observed a wide angular acceptance of light over $\theta=0^{\circ}-80^{\circ}$. This demonstration opens a new door for light trapping and near-unity absorption over broad $\lambda$ s and wide $\theta$ s using photoniccrystal thin film architecture.

S. Y. L. gratefully acknowledges financial support from DOE-BES under award DE-FG02-06ER46347. M. L. H. acknowledges partial financial support from NSC 101-2221E-009-110 and also travel support from DOE-BES for international collaboration. S. J. acknowledges support from the U.S. Department of Energy under contract DE-FG02-10ER46754.

\section{References}

1. W. Shockley and H. J. Queisser, J. Appl. Phys. 32, 510 (1961).

2. M. Planck, The Theory of Heat Radiation (Dover, 1912).

3. E. Yablonovitch, J. Opt. Soc. Am. 72, 899 (1982).

4. P. Bermel, C. Luo, L. Zeng, L. C. Kimerling, and J. D. Joannopoulos, Opt. Express 15, 16986 (2007).

5. D. M. Schaadt, B. Feng, and E. T. Yu, Appl. Phys. Lett. 86, 063106 (2005).

6. V. E. Ferry, M. A. Verschuuren, H. B. T. Li, E. Verhagen, R. J. Walters, R. E. I. Schropp, H. A. Atwater, and A. Polman, Opt. Express 18, A237 (2010).

7. S. B. Mallick, M. Agrawal, and P. Peumans, Opt. Express 18, 5691 (2010).

8. J. Zhu, Z. Yu, G. F. Burkhard, C. M. Hsu, S. T. Connor, Y. Xu, Q. Wang, M. McGehee, S. Fan, and Y. Cui, Nano Lett. 9, 279 (2009).

9. Q. Lin, B. Hua, S. Leung, X. Duan, and Z. Fan, Nano Lett. 7, 2725 (2013).

10. J. M. Gee, in 29th IEEE Photovoltaic Specialists Conference (IEEE, 2002), p. 150.

11. D. Zhou and R. Biswas, J. Appl. Phys. 103, 093102 (2008).

12. Y. Park, E. Drouard, O. E. Daif, X. Letartre, P. Viktorovitch, A. Fave, A. Kaminski, M. Lemiti, and C. Seassal, Opt. Express 17, 14312 (2009).

13. M. L. Bozzola and L. C. Andreani, Opt. Express 20, A224 (2012).

14. S. Y. Lin, V. M. Hietala, L. Wang, and E. D. Jones, Opt. Lett. 21, 1771 (1996).

15. H. Kosaka, T. Kawashima, A. Tomita, M. Notomi, T. Tamamura, T. Sato, and S. Kawakami, Phys. Rev. B 58, R10096 (1998).

16. A. Chutinan and S. John, Phys. Rev. A 78, 23825 (2008).

17. G. Demésy and S. John, J. Appl. Phys. 112, 074326 (2012).

18. A. Deinega and S. John, J. Appl. Phys.112, 074327 (2012).

19. J. G. Fleming, S. Y. Lin, I. El-Kady, R. Biswas, and K. M. Ho, Nature 417, 52 (2002).

20. S. Y. Lin, J. G. Fleming, R. Lin, M. M. Sigalas, and K. M. Ho, J. Opt. Soc Am. B 18, 32 (2001).

21. Y. J. Hung, S. L. Lee, B. J. Thibeault, and L. A. Coldren, J. Sel. Top. Quan. Elec. 17, 869 (2011).

22. J.-Q. Xi, M. F. Schubert, J. K. Kim, E. F. Schubert, M. Chen, S. Y. Lin, W. Liu, and J. A. Smart, Nat. Photonics 1, 176 (2007)

23. Y. F. Chao, K. Y. Lee, and Y. D. Lin, Appl. Opt. 45, 3935 (2006).

24. S. Kageyama, M. Akagawa, and H. Fujiwara, Phys. Rev. B 83, 195205 (2011).

25. M.-L. Kuo, D. J. Poxson, Y. S. Kim, F. W. Mont, J. K. Kim, E. F. Schubert, and S. Y. Lin, Opt. Lett. 33, 2527 (2008).

26. T. Suezaki, J. I. L. Chen, T. Hatayama, T. Fuyuki, and G. A. Ozin, Appl. Phys. Lett. 96, 242102 (2010). 